\title{
Conhecimento dos enfermeiros residentes sobre leitura e registro do indicador químico nos processos de esterilização de produtos para a saúde
}

Knowledge of resident nurses on reading and recording the chemical indicator in the sterilization processes of health products

Conocimiento de las enfermeras residentes en la lectura y registro del indicador químico en los procesos de esterilización de productos sanitarios

Thamires Nunes da Silva Lima

ORCID: https://orcid.org/0000-0001-9326-7748 Universidade Federal do Estado do Rio de Janeiro, Brasil

E-mail: thami.n.lima@hotmail.com

Tatiana de Gouvêa Martins

ORCID: https://orcid.org/0000-0002-3259-1891 Universidade Federal do Estado do Rio de Janeiro, Brasil E-mail: tgouveam@hotmail.com

Luiz Henrique da Silva Inácio ORCID: https://orcid.org/0000-0001-6639-8312 Universidade Federal do Estado do Rio de Janeiro, Brasil E-mail: ricoinacio@hotmail.com

Zaíne Melo de Oliveira

ORCID: https://orcid.org/0000-0002-5316-7619 Universidade Federal do Estado do Rio de Janeiro, Brasil E-mail: zainemelo.enf@gmail.com

Ana Karoliny Costa Barbosa ORCID: https://orcid.org/0000-0003-0240-911X Universidade Federal do Estado do Rio de Janeiro, Brasil E-mail: karolbarbosajf@gmail.com

Raquel Calado da Silva Gonçalves ORCID: https://orcid.org/0000-0003-0158-5031 Ministério da Saúde, Brasil E-mail: raquelcalado@yahoo.com.br Aline Coutinho Sento Sé ORCID: https://orcid.org/0000-0001-9301-0379 Ministério da Saúde, Brasil E-mail: aline2506@hotmail.com

Teresa Tonini

ORCID: https://orcid.org/0000-0002-5253-2485 Universidade Federal do Estado do Rio de Janeiro, Brasil E-mail: ttonini@ terra.com.br

\begin{abstract}
Resumo
Objetivo: investigar o conhecimento dos residentes de enfermagem da Universidade Federal do Rio de Janeiro a respeito da realização da leitura e registro do indicador químico utilizado no interior das caixas e pacotes de instrumentais cirúrgicos e produtos para a saúde. Metodologia: estudo descritivo, com abordagem quantitativa, realizado com enfermeiros residentes da Universidade Federal do Estado do Rio de Janeiro. Critérios de inclusão: ser enfermeiro e residente de enfermagem da UNIRIO com matrícula ativa. Utilizou-se um instrumento enviado por correio eletrônico. Os dados coletados foram categorizados e armazenados em planilha eletrônica do sistema Excel®. O perfil profissional da amostra foi descrito por frequências absolutas e relativas das variáveis categóricas. Calculouse a proporção de concordância dos enfermeiros residentes, no que diz respeito às afirmativas apresentadas no instrumento para análise deles. (CAAE: 39525820.7.0000.5285). Resultados: responderam ao questionário 43 enfermeiros, com idade entre 25 e 28 anos, maioria do sexo feminino e formados entre 1 e 5 anos. Observou-se que 74\% dos enfermeiros residentes consideraram-se capazes de realizar a leitura do indicador químico. Conclusão: conclui-se que os enfermeiros residentes possuem conhecimento e segurança para avaliar o resultado de um indicador químico tipo 5 ou 6 e sentem-se aptos a avaliá-lo.
\end{abstract}

Palavras-chave: Enfermagem; Esterilização; Infecção da ferida cirúrgica; Educação de pós-graduação em enfermagem; Enfermagem baseada em evidências; Ensino. 


\begin{abstract}
Objective: to investigate the knowledge of nursing residents at the Federal University of Rio de Janeiro regarding the reading and recording of the chemical indicator used inside the boxes and packages of surgical instruments and health products. Methodology: a descriptive study, with a quantitative approach, carried out with resident nurses at the Federal University of the State of Rio de Janeiro. Inclusion criteria: being a nurse and nursing resident at UNIRIO with active enrollment. An instrument sent by electronic mail was used. The collected data were categorized and stored in an Excel® spreadsheet. The professional profile of the sample was described by absolute and relative frequencies of categorical variables. The proportion of agreement of resident nurses was calculated, with regard to the statements presented in the instrument for their analysis. (CAAE: 39525820.7.0000.5285). Results: 43 nurses answered the questionnaire, aged between 25 and 28 years, mostly female and graduated between 1 and 5 years. It was observed that $74 \%$ of resident nurses considered themselves capable of reading the chemical indicator. Conclusion: it is concluded that resident nurses have the knowledge and security to evaluate the result of a chemical indicator type 5 or 6 and feel able to evaluate it.
\end{abstract}

Keywords: Nursing; Sterilization; Surgical wound infection; Education, nursing, graduate; Evidence-based nursing; Teaching.

\title{
Resumen
}

Objetivo: investigar los conocimientos de los residentes de enfermería de la Universidad Federal de Río de Janeiro sobre la lectura y registro del indicador químico utilizado dentro de las cajas y paquetes de instrumental quirúrgico y productos de salud. Metodología: estudio descriptivo, con abordaje cuantitativo, realizado con enfermeras residentes de la Universidad Federal del Estado de Rio de Janeiro. Criterios de inclusión: ser enfermero y residente de enfermería en UNIRIO con matrícula activa. Se utilizó un instrumento enviado por correo electrónico. Los datos recopilados se categorizaron y almacenaron en una hoja de cálculo de Excel®. El perfil profesional de la muestra se describió mediante frecuencias absolutas y relativas de variables categóricas. Se calculó la proporción de concordancia de enfermeras residentes, con respecto a los enunciados presentados en el instrumento para su análisis. (CAAE: 39525820.7.0000.5285). Resultados: respondieron el cuestionario 43 enfermeras, con edades entre 25 y 28 años, en su mayoría mujeres y egresadas entre 1 y 5 años. Se observó que el $74 \%$ de las enfermeras residentes se consideraba capaz de leer el indicador químico. Conclusión: se concluye que las enfermeras residentes tienen el conocimiento y la seguridad para evaluar el resultado de un indicador químico tipo 5 o 6 y se sienten capaces de evaluarlo.

Palabras clave: Enfermería; Esterilización; Infección de la herida quirúrgica; Educación de postgrado en enfermeira; Enfermería basada en la evidencia; Ensenãnza.

\section{Introdução}

O Centro de Material e Esterilização (CME) é uma unidade de suporte dentro da instituição de saúde responsável pelas etapas do processo de esterilização: a limpeza, a inspeção, o preparo, a esterilização, o armazenamento e a distribuição de materiais (SOBECC, 2017).

Segundo a Organização Mundial de Saúde (OMS), o principal objetivo do CME é fornecer produtos para saúde (PPS) reprocessados de forma segura para procedimentos realizados nas unidades de saúde, áreas de cuidado crítico, cirurgia ambulatorial, nutrição, diálise e endoscopia (OMS, 2016).

Com a finalidade de prevenir Infecções Relacionadas à Saúde (IRAS), o processo de esterilização visa à destruição de microrganismos existentes em artigos hospitalares. Elas podem ser prevenidas por meio da esterilização e descontaminação de instrumentos cirúrgicos e PPS (OMS, 2016; SOBECC, 2017).

O monitoramento da esterilização deve abranger avaliações físicas, químicas e biológicas. Para o controle químico são utilizados diversos tipos de indicadores, com diferentes apresentações no mercado. Um monitoramento adequado é necessário para minimizar o risco de infecção (Basu, 2019). Assim, neste estudo, abordou-se os indicadores tipo 5 e 6.

Os indicadores devem ser projetados para reagir a todas as variáveis críticas do processo de esterilização dentro de um intervalo específico de ciclos de esterilização (SOBECC, 2017).

O indicador químico tipo 5 é composto por uma tinta termo crômica que altera a cor e/ ou forma reagindo às condições do processo, oferecendo resposta através de uma nítida mudança de coloração, de acordo com especificações do fabricante. Como controle interno de avaliação do vapor esterilizante, o indicador químico deve ser inserido no interior das 
caixas e pacotes a serem esterilizados (SOBECC, 2017).

A leitura e avaliação do indicador químico contido dentro das caixas e pacotes dos produtos para a saúde esterilizados requer raciocínio clínico, tomada de decisão e conhecimento dos profissionais de saúde, contribuindo com a segurança do paciente (Gonçalves \& Santana, 2013).

Os resultados e informações gerais devem ser registrados e arquivados, assegurando a rastreabilidade, em conformidade à legislação específica e as exigências da inspeção sanitária (Brasil, 2012).

Consequentemente, a correta utilização, leitura e avaliação do indicador químico, permitem a rastreabilidade das etapas do processamento dos PPS, além de contribuir com programas de prevenção e controle de eventos adversos, segurança do paciente e controle de infecção em serviços de saúde.

O estudo tem como objeto a avaliação do conhecimento dos enfermeiros residentes sobre o uso indicador químico, contido nas caixas e pacotes de instrumentais cirúrgicos e produtos para saúde, processados no CME.

Procedimentos adequados e boas práticas de processamento de PPS têm sido enfatizados em publicações que abordam a segurança do paciente na prevenção das IRAS. Boas práticas são fundamentais para controle das IRAS, constituindo-se imprescindíveis para uma assistência segura e de qualidade, através de medidas preventivas, correção de falhas e adoção de protocolos institucionais (Moriya \& Takeiti, 2016).

Neste contexto estão inseridos os residentes de enfermagem durante treinamento em serviço no CME, desenvolvendo atividades que compõem o processo de esterilização de artigos para a saúde.

A residência em enfermagem apresenta-se como uma modalidade de ensino e capacitação profissional para o mercado de trabalho, sendo de grande importância que a instituição de saúde propicie condições favoráveis de aprendizagem com vistas à qualidade do cuidado ofertado (Oliveira, Carvalho, Teixeira, Zeitoune, Sabóia \& Gallasch, 2017).

Compreender os requisitos de boas práticas para o processamento de PPS é fundamental para a atuação do enfermeiro, pois garante a segurança do paciente e dos profissionais envolvidos no cuidado, assim como o controle de eventos adversos e de infecção em serviços de saúde (Brasil, 2012).

Assim, definiu-se como objetivo do estudo: investigar o conhecimento dos residentes de enfermagem da Universidade Federal do Estado do Rio de Janeiro a respeito da realização da leitura e registro do indicador químico utilizado no interior das caixas e pacotes de instrumentais cirúrgicos e produtos para a saúde.

\section{Metodologia}

Trata-se de um estudo descritivo, com abordagem quantitativa, realizado com os enfermeiros residentes de enfermagem da Universidade Federal do Estado do Rio de Janeiro (UNIRIO), através da aplicação de um formulário. A coleta dos dados realizou-se no período de 11 de dezembro de 2020 a 18 de janeiro de 2021, após aprovação do Comitê de Ética em Pesquisa (CEP) da UNIRIO (CAAE: 39525820.7.0000.5285).

A pesquisa quantitativa é aquela em que se coletam e analisam dados quantitativos sobre variáveis. Dessa forma, este tipo de pesquisa é capaz de identificar a natureza profunda das realidades, seu sistema de relações, sua estrutura dinâmica. Ela também pode determinar a força de associação ou correlação entre variáveis, a generalização e objetivação dos resultados através de uma mostra que faz inferência a uma população. Além do estudo da associação ou correlação, a pesquisa quantitativa também pode, ao seu tempo, fazer inferências causais que explicam por que as coisas acontecem ou não de uma forma determinada (Esperon, 2017).

Neste contexto, as pesquisas de abordagem quantitativa realizadas na internet podem ser desenvolvidas através de enquetes e questionários virtuais. Facilita bastante o trabalho do pesquisador pela praticidade que lhe é característica, reduzindo custos e o uso do recurso tempo, dando capacidade de acesso a um grande número de pessoas, diminuindo 
distâncias, dando opções de movimentos ou não à pesquisa e facilitando a edição dos dados coletados (Camboim, Bezerra \& Guimarães, 2015).

Considerou-se como critérios de inclusão: ser enfermeiro e residente de enfermagem da UNIRIO com matrícula ativa. Excluíram-se os residentes de enfermagem do primeiro ano, tendo em vista que a maioria deles ainda não desenvolveu nenhum tipo de atividade prática no setor de CME durante a realização da residência. Assim, foram abordados 82 enfermeiros, sendo 02 excluídos por desistência da residência.

Utilizou-se um instrumento enviado por correio eletrônico, disponibilizado pelos autores e avaliado por meio de uma escala Likert. Os enfermeiros pontuaram conforme o seu julgamento, da seguinte forma: 1 - discordo totalmente, 2- discordo, 3 - indiferente, 4 - concordo e 5 - concordo totalmente.

$\mathrm{Na}$ escala Likert, os participantes elegem um número de 1 a 5 relacionado com o grau de concordância em relação à afirmação. O número 1 corresponde à total discordância e o número 5 à total concordância em relação à afirmação completa (Pereira, Shitsuka, Parreira \& Shitsuka, 2018).

Os dados coletados foram categorizados e armazenados em planilha eletrônica do sistema Excel®. O perfil profissional da amostra foi descrito por frequências absolutas e relativas das variáveis categóricas.

Em relação à análise, a escala Likert foi recodificada de forma dicotômica, consederando-se as pontuações 1, 2 e 3 inadequadas e os itens pontuados com 4 e 5, considerou-se adequados. Após, calculou-se a proporção de concordância dos enfermeiros residentes, no que diz respeito às afirmativas apresentadas no instrumento para análise deles.

Os enfermeiros foram abordados pelos pesquisadores através de correio eletrônico, com garantia do sigilo, da privacidade, convidados a participar da pesquisa e assinar o Termo de Consentimento Livre e Esclarecido (TCLE). Com o retorno do TCLE preenchido e assinado, encaminhou-se o instrumento por correio eletrônico.

Em relação aos riscos da pesquisa, admitiu-se um risco mínimo, pois a resposta a algum questionamento poderia gerar ansiedade no participante além do desprendimento de tempo em responder ao instrumento.

\section{Resultados e Discussão}

Responderam ao questionário 43 enfermeiros, representando 53,75\% da participação prevista. A tabela 1 apresenta a distribuição das características sóciodemográficas e formação profissional dos participantes do estudo. 
Tabela 1 - Distribuição dos enfermeiros, segundo as características sóciodemográficas e de formação profissional. Rio de Janeiro, $2021(n=43)$.

\begin{tabular}{|c|c|c|}
\hline Variáveis & Frequência & $\begin{array}{c}\text { Frequência relativa } \\
\qquad(\%)\end{array}$ \\
\hline \multicolumn{3}{|l|}{ Idade (anos) } \\
\hline$[22,25[$ & 3 & 6,98 \\
\hline$[25,28[$ & 18 & 41,86 \\
\hline$[28,31[$ & 13 & 30,23 \\
\hline$[31,34[$ & 5 & 11,63 \\
\hline$[34,37[$ & 1 & 2,32 \\
\hline$[37,40[$ & - & - \\
\hline$[40,43[$ & 1 & 2,32 \\
\hline$[43,46[$ & 2 & 4,65 \\
\hline Total & 43 & 100 \\
\hline \multicolumn{3}{|l|}{ Sexo } \\
\hline Feminino & 36 & 83,72 \\
\hline Masculino & 7 & 16,28 \\
\hline Total & 43 & 100 \\
\hline
\end{tabular}

Fonte: Autores.

Observa-se na Tabela 1 que a maioria dos participantes tem entre 25 e 28 anos de idade, são do sexo feminino e com tempo de formação de 1 a 5 anos.

Os resultados relacionados ao conhecimento dos enfermeiros a respeito da realização da leitura e registro do indicador químico utilizado no interior das caixas e pacotes de instrumentais cirúrgicos e PPS, encontram-se registrados na Tabela 2. 
Tabela 2 - Conhecimento dos enfermeiros a respeito da realização da leitura e registro do indicador químico utilizado no interior das caixas e pacotes de instrumentais cirúrgicos e PPS, Rio de Janeiro, 2021 (n=43).

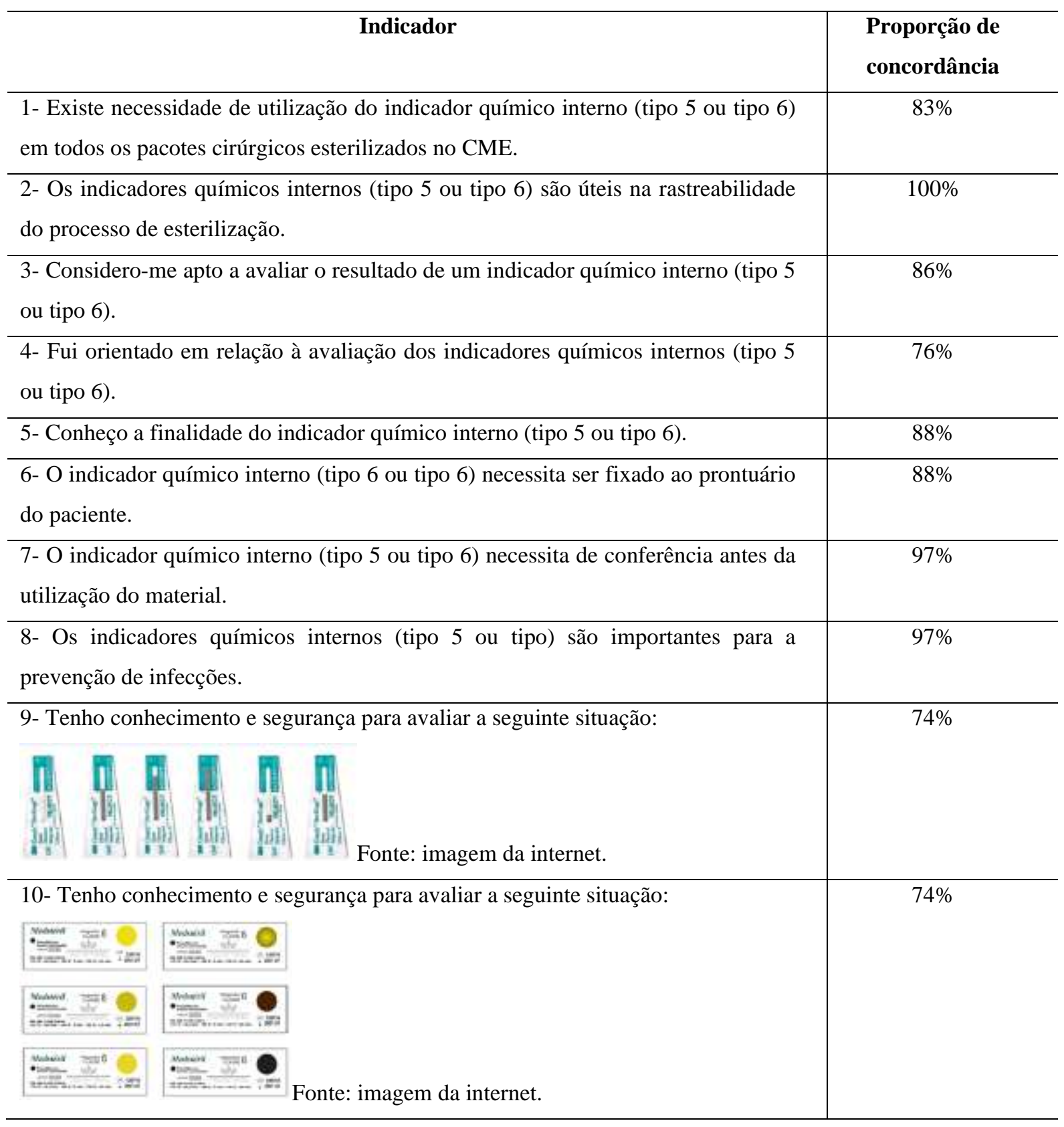

Fonte: Autores.

A RDC 15 (2012), em seu artigo 96, preconiza a utilização de indicadores tipo 5 ou 6 no monitoramento do processo de esterilização. Neste estudo, todos os enfermeiros foram unânimes em afirmar que os indicadores químicos internos (tipo 5 ou tipo 6) são úteis na rastreabilidade do processo de esterilização, o que mostra o entendimento da importância de sua utilização.

Neste estudo, 74\% dos enfermeiros residentes consideraram-se capazes de realizar a leitura do indicador químico. Pesquisas apontam que alguns enfermeiros não apresentam este estímulo em buscar o conhecimento. As instituições de ensino superior, geralmente não abordam de forma incisiva os conteúdos relacionados à prática profissional em CME, ficando este à cargo do campo prático (Lucon, Bracialli, Pirolo \& Munhoz, 2017).

Os indicadores químicos reagem por mudança termo crômica quando expostos ao processo de esterilização. Possuem 
a finalidade de controle do pacote esterilizado, tanto interna quanto externamente, contribuindo com a rastreabilidade do material processado. São essenciais para assegurar a qualidade do processo de esterilização, monitorando falhas. (Oliveira, Silva, 2015; OMS, 2016; Quintino, 2017; Link, 2019; Gonçalves, Sé, Pezzi, Pinto, Souza, Fernández, Hernández \& Tonini, 2020).

A "Falha no indicador químico após a esterilização" constitui-se um fator de risco para contaminação de PPS, sendo recomendada a verificação de sua condição (leitura) após o processo de esterilização (Gonçalves \& Santana, 2013; Gonçalves et al, 2020).

A identificação de falhas no indicador químico após o processamento pelo enfermeiro é precedida da adoção de medidas corretivas e investigação dos motivos que levaram ao resultado insatisfatório (Link, 2019; Laranjeira, Bronzatti, Bruna, Souza, Graziano \& Lusignan, 2020).

Boas práticas recomendam a utilização de um indicador interno no interior das caixas cirúrgicas e pacotes. Ele deve ser posicionado na área considerada de maior desafio à penetração do agente esterilizante (SOBECC, 2017; AORN, 2017).

A falha de um indicador químico interno compromete a segurança do processo de esterilização da caixa cirúrgica ou do pacote. Recomenda-se a não utilização e o reprocessamento do PPS nestes casos (SOBECC, 2017; AORN, 2017).

$\mathrm{O}$ trabalho realizado em CME ainda carece de visibilidade. Na prática, muitos profissionais não possuem o conhecimento específico exigido para desempenhar as atividades no setor (Sanchez, Silveira, Figueiredo, Mancia, Schwoncke \& Gonçalves, 2018; Ribeiro, Amaral, Souza, Silva, Brito, Negrão, Pompeu \& Pedrosa, 2019).

Ressalta-se que as atividades de enfermagem em CME estão diretamente relacionadas com o processo de controle de infecção. O processamento de PPS sem a observância de boas práticas alinhadas ao conhecimento científico contribui para infecções, pondo em risco a segurança do paciente (Oliveira, Silva, 2015; Gonçalves, Santana, Silvino, Christovam, Pereira \& Schultz, 2015; Ribeiro et al, 2019).

O número reduzido da amostra caracteriza-se como uma limitação do estudo. Destaca-se que este estudo foi realizado com uma população específica, podendo não refletir a realidade de outras instituições de ensino.

\section{Conclusão}

Conclui-se que os enfermeiros residentes possuem conhecimento e segurança para avaliar o resultado de um indicador químico tipo 5 ou 6 e sentem-se aptos a avalia-lo. Além disso, entendem ser necessária a sua utilização em todos os pacotes e caixas cirúrgicas processadas, bem como a sua importância no processo de rastreabilidade do material processado.

Faz-se necessária a abordagem dos conteúdos relacionados à CME, não apenas na residência (campo prático), mas principalmente durante a graduação, tendo em vista a formação generalista do enfermeiro.

Outros estudos podem contribuir com a temática em questão, abordando a realidade de diferentes instituições de ensino e que envolvam assuntos diversos relacionados às etapas de processamento de PPS.

\section{Referências}

Associação Brasileira de Enfermeiros de Centro Cirúrgico, Recuperação Anestésica e Centro de Material e Esterilização (SOBECC). (2017) Diretrizes de práticas em enfermagem cirúrgica e processamento de produtos para a saúde. (7a ed.). SOBECC.

Association of periOperative Registered Nurses (AORN). (2017) Guidelines for cleaning and care of surgical instruments. http://www.nascecme.com.br/assinante/GUIDE LINE_FOR_CLEANING_AND_CARE_OF_SURGICAL_INSTRUMENTS.pdf

Basu, D. (2019) Requirement of hollow process challenge device for monitoring hollow and complex instruments sterilization: a simulator for proper sterility assurance. Infection Control \& Hospital Epidemiology, 40(8), 951-952. https://doi.org/10.1017/ice.2019.138

Brasil (2012). Ministério da Saúde. Agência Nacional de Vigilância Sanitária (ANVISA). Resolução da diretoria colegiada - RDC nº 15, de 15 de março de 2012. Dispõe sobre requisitos de boas práticas para o processamento de produtos para saúde e dá outras providências. https://bvsms.saude.gov.br/bvs/saudelegis/anvisa/2012/rdc0015_15_03_2012.html 
Camboim, L. G., Bezerra, E. P., Guimarães, I. J. B. (2015) Pesquisando na internet: uma análise sobre metodologias utilizadas em dissertações de mestrado do programa de pós graduação em ciência da informação da UFPB. Biblionline, 11 (2), 123-34. https://periodicos.ufpb.br/ojs 2/index.php/biblio/article/view/25380

Esperon, J. M. T. (2017) Pesquisa quantitativa na ciência da enfermagem. Escola Anna Nery, 21 (1), e20170027. https://doi.org/10.5935/14148145.20170027

Gonçalves, R. C. S. \& Santana, R. F. (2013). Risco para contaminação de artigos e materiais: uma proposta de diagnóstico de enfermagem. Revista de Enfermagem UFPE on line, 7 (3), 1059-1063. https://periodicos.ufpe.br/revistas/revistaenfermagem/article/view/11577/13584

Gonçalves, R. C. S, Santana, R. F., Silvino, Z. R., Christovam, B. P., Pereira, P. O. \& Schultz. (2015) Prática operacional do enfermeiro no centro de material e esterilização: revisão integrativa. Revista de enfermagem UFPE on line, 9(2), 745-749.

Gonçalves, R. C. S., Sé, A. C. S., Pezzi, M. C. S., Pinto, C. M. I., Souza, P. A., Fernández, B. M., Hernández, P. E., \& Tonini, T. (2020). Atividades de enfermagem para o diagnóstico "Risco para contaminação de artigos". Research, Society and Development, 9(9), e683997794. https://doi.org/10.33448/rsdv9i9.7794

Laranjeira, P. R., Bronzatti, J. A. G., Bruna, C. Q. M., Souza, R. Q., Graziano, K. U., \& Lusignan, V. (2020). False positive results of Bowie and Dick type test used for hospital steam sterilizer with slower come-up ramps: a case study. PLoS One, 15(1), e0227943.

Ling, M.L., Ching, P., Widitaputra, A., Stewart A., Sirijindadirat, N. \& Thu, L.T.A. (2018) APSIC guidelines for disinfection and sterilization of instruments in health care facilities. Antimicrobial Resistance \& Infection Control, 7(25). https://doi.org/10.1186/s13756-018-0308-2

Link, T. (2019) Guideline implementation: sterilization. AORN Journal, 109(6), 772-82. https://doi.org/10.1002/aorn.12668

Lucon, S. M. R., Bracialli, L. A. D., Pirolo, S. M. \& Munhoz, C. C. (2017). Formação do enfermeiro para atuar na central de esterilização. Revista SOBECC, 22(2), 90-97.

Moriya, G. A. A. \& Takeiti, M. H. (2016). O trabalho da enfermagem em centro de material e esterilização e sua implicação para a segurança do paciente. Revista SOBECC, 21(1), 1-2.

Organização Mundial de Saúde (OMS). (2016). Descontaminação e reprocessamento de produtos para a saúde em instituições de assistência à saúde. http://nascecme.com.br/descontaminacao-e-reprocessamento-de-produtos-para-saude-em-instituicoes-de-assistencia-a-saude/

Oliveira, A. C., \& Silva, M. V. G. (2015). Teoria e prática na prevenção da infecção do sítio cirúrgico. Manole.

Oliveira, E. B., Carvalho, R. A., Teixeira, E., Zeitoune, R. C. G., Sabóia, V. M. \& Gallasch, C. H. (2017). Fatores intervenientes na formação de enfermeiros residentes: visão de egressos de um programa de residência. Revista Mineira de Enfermagem, 21, e-1064. http://www.dx.doi.org/10.5935/14152762.20170074

Pereira, A. S., Shitsuka, D. M., Parreira, F. J. \& Shitsuka, R. (2018) Metodologia da pesquisa científica. https://repositorio.ufsm.br/bitstream/handle/1/15824/Lic_Computacao_Metodologia-Pesquisa-Cientifica.pdf?sequence=1

Quintino, A. P. N. N. (2017) Testes desafio em cargas subsequentes na esterilização a vapor saturado: estudo comparativo dos desempenhos. Revista SOBECC, 22(3),145-151.

Ribeiro, J. M. S., Amaral, C. A., Souza, J. P., Silva, S. L. B., Brito, R. G., Negrão, M. S. O., Pompeu, J. S. \& Pedrosa, L. T. J. S. (2019). Avaliação do conhecimento da equipe de enfermagem no processo de esterilização de materiais cirúrgicos. Revista Eletrônica Acervo Saúde, 11(15), 1-9. https://doi.org/10.25248/reas.e1177.2019

Sanchez, M. L., Silveira, R. S., Figueiredo, P. P., Mancia, J. R., Schwonke, C. R. G. B. \& Gonçalves, N. G. C. (2018) Estratégias que contribuem para a visibilidade do trabalho do enfermeiro na central de material e esterilização. Texto \& Contexto Enfermagem, 27 (1), 1-9. 